\title{
Efficacy of submucosal injection of different solutions inclusive blood components on mucosa elevation for endoscopic resection
}

This article was published in the following Dove Press journal:

Clinical and Experimental Gastroenterology

16 April 2012

Number of times this article has been viewed

\author{
Oliver H Al-Taie' \\ Yildiz Bauer ${ }^{2}$ \\ Christoph G Dietrich ${ }^{3}$ \\ Wolfgang Fischbach ${ }^{2}$ \\ 'Department of Internal Medicine, \\ Sankt Elisabeth-Hospital, Gütersloh, \\ ${ }^{2}$ Department of Internal Medicine II, \\ Klinikum Aschaffenburg, \\ Aschaffenburg, ${ }^{3}$ Department of \\ Internal Medicine, Bethlehem- \\ Hospital, Stolberg, Germany
}

Background: Endoscopic resection has become the standard treatment for noninvasive gastrointestinal malignancies. In flat mucosal tumors, normal saline is frequently used for submucosal fluid injection in order to reduce the risk of complications during endoscopic resection. Recent studies have demonstrated longer-lasting mucosa elevation by injection of agents such as hyaluronic acid or glyceol, rather than normal saline. We investigated the efficacy of different blood components in comparison with other solutions for use as a submucosal fluid cushion.

Methods: Normal saline, sodium hyaluronate, glyceol, hydroxyethyl starch, serum, plasma, and whole blood were evaluated for their effectiveness in creating a submucosal cushion. One milliliter of each solution was injected into the submucosa of $5 \times 5 \mathrm{~cm}$ specimens of resected porcine stomach. Mucosa elevation was measured before and up to 60 minutes after injection. Results: The shortest duration of mucosa elevation was observed after injection of normal saline, glyceol, and $0.125 \%$ hyaluronic acid. A significantly longer duration was obtained after injection of hydroxyethyl starch, $0.25 \%$ and $0.5 \%$ hyaluronic acid, serum, and plasma. However, whole blood generated a longer-lasting mucosa elevation than all other agents.

Conclusion: The results of the current study suggest that whole blood is more effective in generating long-lasting mucosa elevation than any other commonly used solution. Because autologous blood is readily available at almost no cost, this seems to be an optimal agent for creating the mucosa elevation needed for endoscopic resection. Further in vivo studies in humans are needed to clarify the potential role of autologous blood for long-lasting endoscopic mucosa resection or endoscopic submucosal dissection.

Keywords: submucosal injection, blood components, sodium hyaluronate, glycerol, normal saline, endoscopic mucosal resection

\section{Introduction}

Endoscopic resection modalities such as endoscopic mucosa resection (EMR) or endoscopic submucosal dissection (ESD) are now established as the standard treatment modality in the treatment of noninvasive mucosal tumors along the gastrointestinal tract. ${ }^{1-5}$ Especially in flat or nonpedunculated lesions, submucosal injection of normal saline (NS) solution is widely used for separating the lesion from the muscularis propria to allow its resection and prevent perforation and thermal injury to the intestinal wall. ${ }^{6-9}$ However, mucosa elevation is usually seen for only a short period after NS injection, so repeated injections are required. In recent years, several solutions, such as sodium hyaluronate, hydroxypropyl methylcellulose, mannitol, glyceol $(\mathrm{G})$, and fibrinogen, have been investigated and have been shown to extend the duration of mucosal elevation. ${ }^{10-14}$ However, disadvantages such as high costs and
Correspondence: Oliver H Al-Taie Department of Internal Medicine, Sankt Elisabeth Hospital, Stadtring Kattenstroth 130, 33332 Gütersloh, Germany Tel +49524I 5077310 Fax +49 524I 5077390 Email oliver.al-taie@sankt-elisabethhospital.de 
difficult administration, as well as concerns about stimulation of tumor cells, have obviated the use of these solutions in routine endoscopy.

The aim of this study was to compare the effectiveness of different components of blood with NS, hyaluronic acid (HA), G, and hydroxyethyl starch (HES) on mucosa elevation after submucosal injection.

\section{Materials and methods}

NS (B Braun Melsungen AG, Melsungen, Germany), 0.125\% HA, $0.25 \%$ HA, 0.5\% HA (Suvenyl ${ }^{\circledR}$, Chugai Pharmaceutical Co, Tokyo, Japan), G (10\% glycerin with $0.9 \% \mathrm{NaCl}$ plus $5 \%$ fructose; Chugai Pharmaceutical Co, Tokyo, Japan), and HES 6\% (Fresenius Kabi AG, Bad Homburg, Germany), as well as whole blood, plasma, and serum (collected from the corresponding author) were used in this study to create a submucosal fluid cushion. Squares of resected porcine stomach $(5 \times 5 \mathrm{~cm})$ were stretched on a corkboard. To ensure a constant distance and angle of the specimens relative to a camera, a special construction with fixed camera position and fixed specimen position was used (Figure 1). One milliliter of one of the solutions was horizontally injected into the submucosa from the margin of the specimen using a $1 \mathrm{~mL}$ syringe with a 23 -gauge needle.

Digital pictures were taken before injection, immediately after injection, and every 5 minutes thereafter for up to 60 minutes - this was controlled by Nikon Camera Control Pro Software (v 1.3.0; Nikon Inc, Melville, NY). Pictures were taken using a Nikon D70S camera with a Nikkor DF Micro $60 \mathrm{~mm}$ zoom lens (Nikon Inc). Each experiment was independently repeated five times. The recorded pictures (Figure 2) were analyzed in a blinded fashion using Adobe Photoshop (v CS3; Adobe Systems Inc, San Jose, CA), which allowed measurement of mucosa height before and after submucosal fluid injection.

Statistical analysis was performed with a Student's $t$-test using GraphPad Prism (v 5.01; GraphPad Software Inc, La Jolla, CA).

\section{Results}

Average mucosa elevation immediately after submucosal injection was between 5.9 and $7.1 \mathrm{~mm}$ (Figure 3). Submucosa injected with whole blood resulted in a slight but significantly higher mucosa elevation compared with G. However, average mucosa elevation did not differ significantly among the other solutions used in this study (Figure 3).

Over time, a decrease of mucosa elevation was observed for all agents used. Significant differences in the duration of

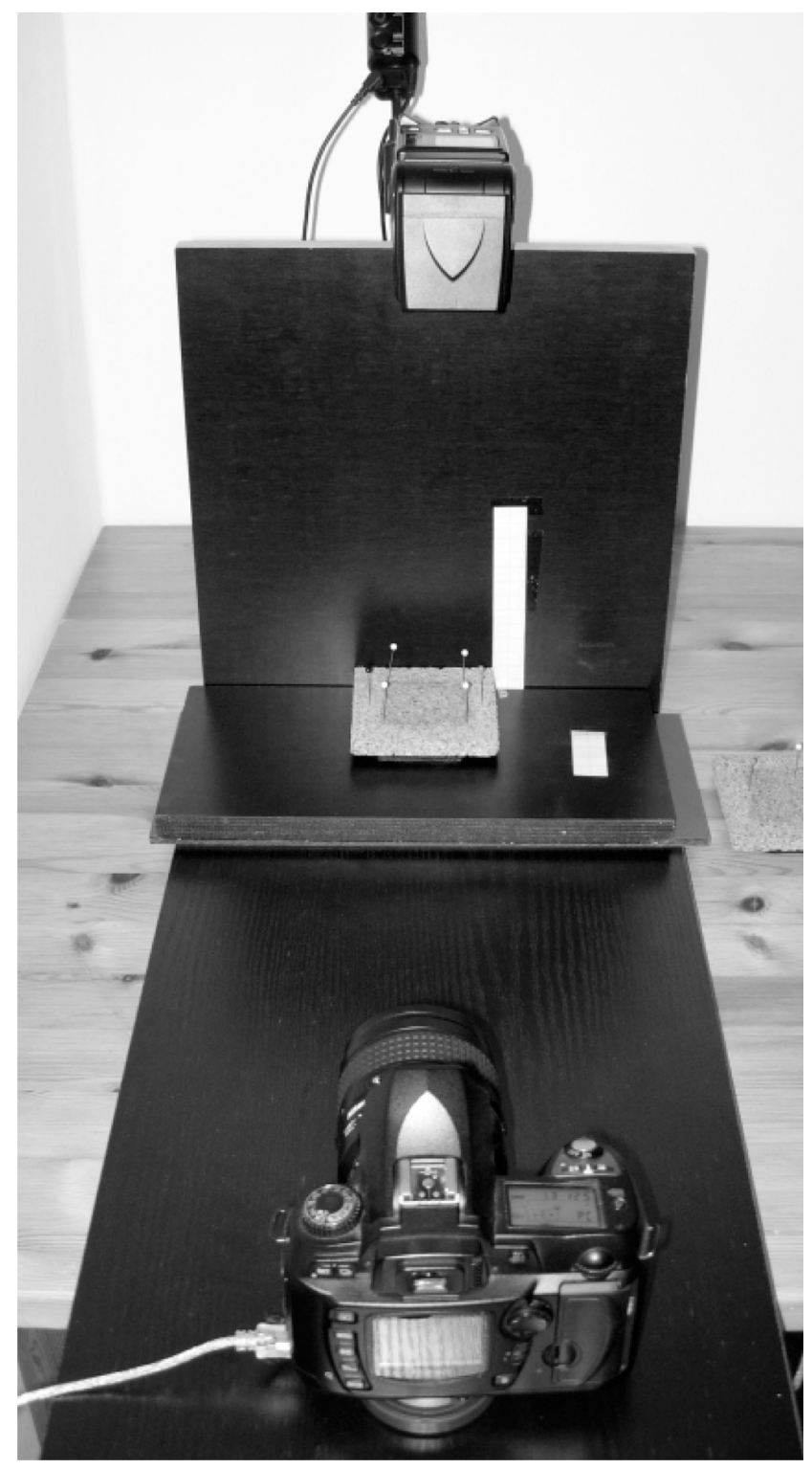

Figure I Test arrangement with fixed position of camera and cork plate.

elevation could be observed and three distinct groups became evident in terms of the rate of decrease and final height plateau. NS, G, and $0.125 \%$ sodium hyaluronate showed the fastest decrease of average mucosa elevation during the observation period, as well as the lowest level of average mucosa elevation after 60 minutes $(3.38-3.55 \mathrm{~mm})$, with no significant difference observed between these solutions. In contrast, serum and plasma produced a significantly slower decrease of average mucosa elevation and a significantly higher final plateau (3.95-4.35 mm), which was comparable to HES, $0.25 \% \mathrm{HA}$, and $0.5 \% \mathrm{HA}$. The slowest decrease of average mucosa elevation and the highest final height plateau $(5.23 \mathrm{~mm})$ was observed by using whole blood (Figures 4 and 5). 


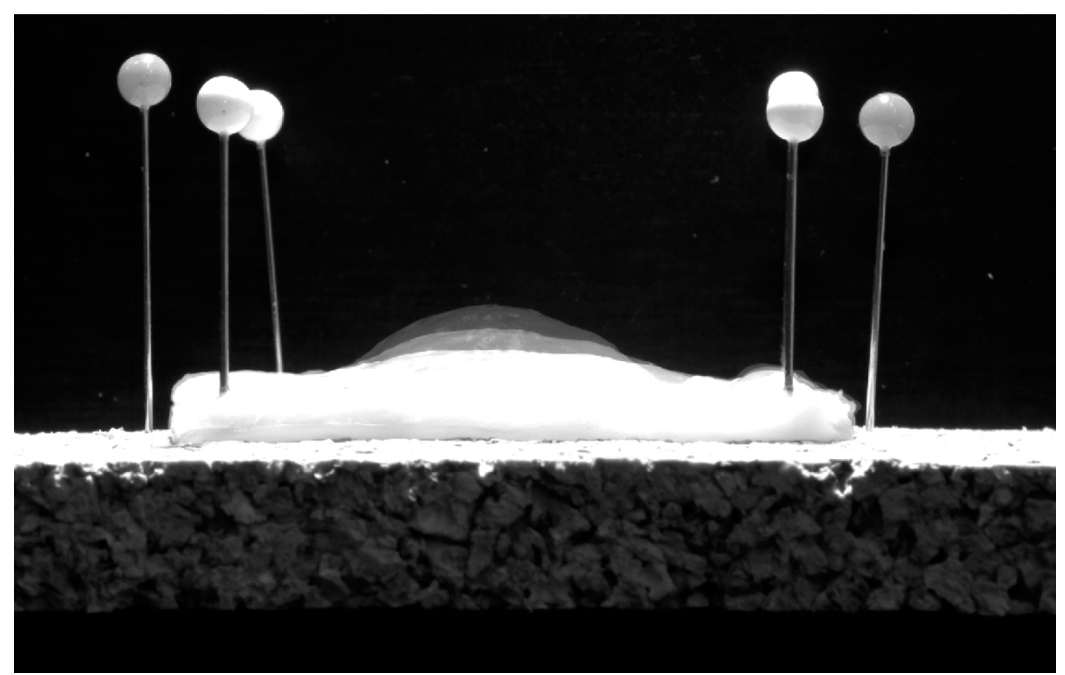

Figure 2 Representative example of overlaid pictures of the gastric wall before, immediately, 5 minutes, and 60 minutes after submucosal fluid injection.

\section{Discussion}

Creating a submucosal cushion is a standard method during endoscopic resection of flat or nonpedunculated lesions in the gastrointestinal tract as this reduces the risk of perforation and thermal injury to the gastrointestinal wall. Particularly for the submucosal dissection technique, mucosa elevation by submucosal fluid injection is considered very important for reducing the risk of perforation. Mucosa elevation is most commonly achieved by injecting NS into the submucosa, but the mucosa elevation this generates often decreases quite rapidly due to diffusion into the surrounding tissue. Therefore, different solutions, such as hyperosmolaric dextrose solutions, hydroxypropyl methylcellulose, $\mathrm{G}$, mannitol, and HA, have been extensively investigated for their ability to lengthen the duration of elevation. ${ }^{10-12,14-17}$

Most notably, HA has been shown to create longerlasting fluid cushions compared with NS both ex vivo and in vivo. However, HA has disadvantages - namely, its high cost and the conflicting data concerning stimulation of tumor growth, which currently inhibit its general use for

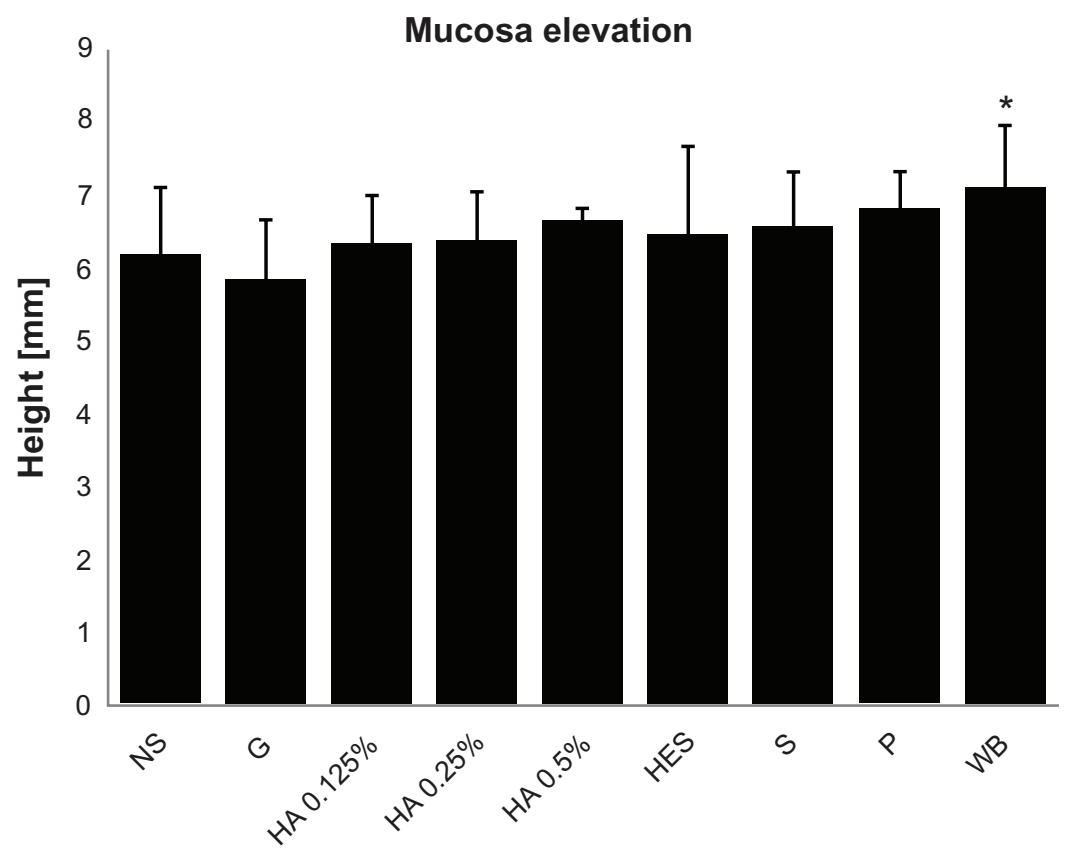

Figure 3 Absolute mucosa elevation immediately after injection of I $\mathrm{mL}$ solution (average height and standard deviation).

Note: $* P<0.05$.

Abbreviations: NS, normal saline; G, glyceol; HA, hyaluronic acid; HES, hydroxyethyl starch; S, serum; P, plasma; WB, whole blood. 


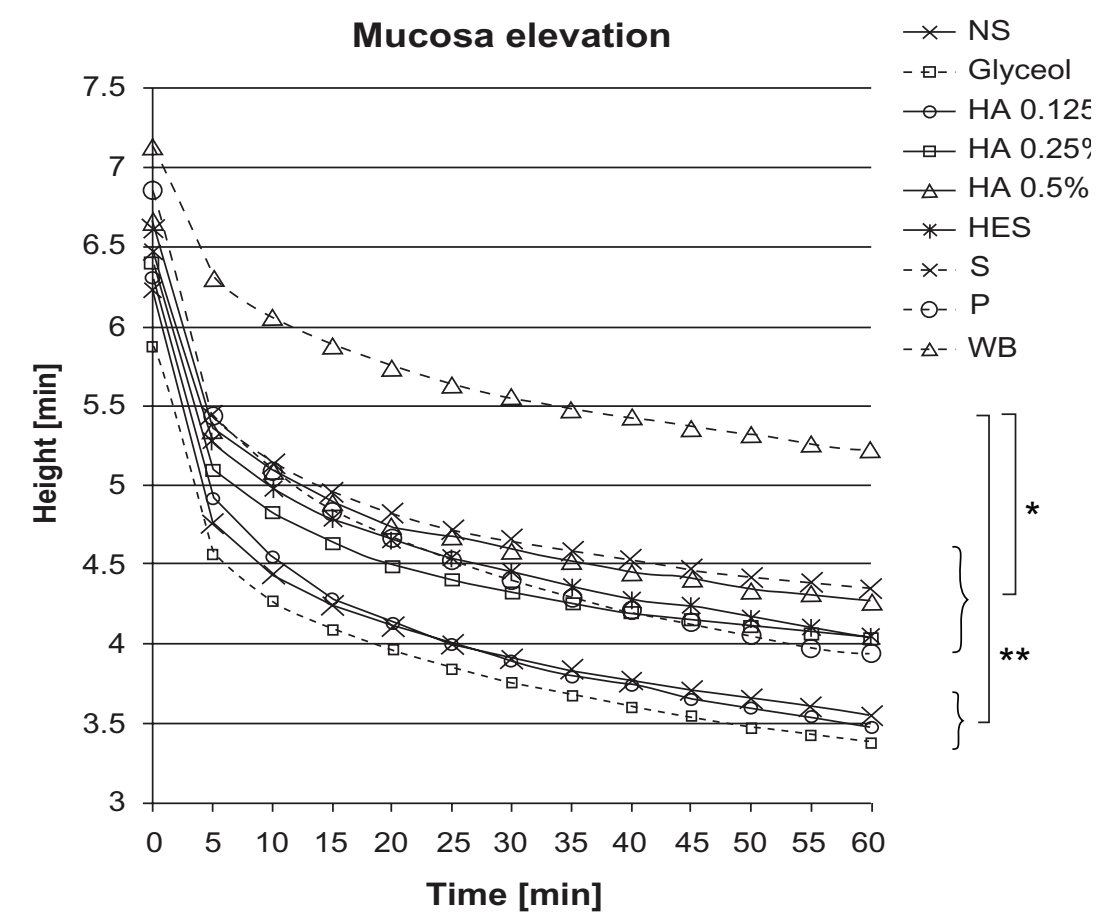

Figure 4 Average absolute mucosa elevation over time after injection of $\mathrm{I} \mathrm{mL}$ solution.

Notes: $* P<0.01$; ${ }^{* *} P<0.0001$.

Abbreviations: NS, normal saline; G, glyceol; HA, hyaluronic acid; HES, hydroxyethyl starch; S, serum; P, plasma; WB, whole blood.

\section{Relative reduction of mucosa elevation}

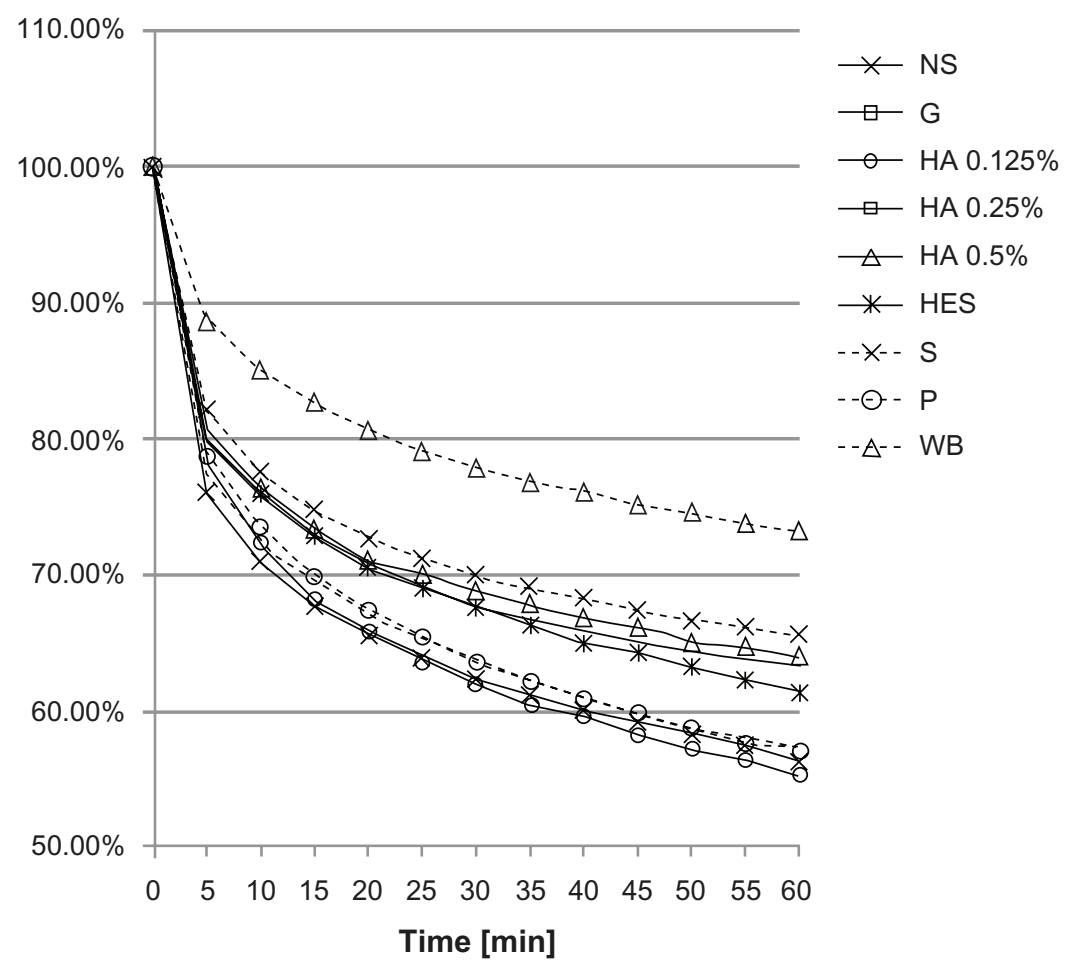

Figure 5 Average relative reduction of mucosa elevation over time after injection of $\mathrm{I} \mathrm{mL}$ solution.

Abbreviations: NS, normal saline; G, glyceol; HA, hyaluronic acid; HES, hydroxyethyl starch; S, serum; P, plasma; WB, whole blood. 
submucosal injection. ${ }^{18,19}$ Hyperosmolaric solutions are implicated in increasing tissue damage and delaying healing of resection ulcers as well as reducing the quality of resected specimens. ${ }^{20}$ Research has demonstrated a correlation between the duration of mucosal elevation and viscosity, but not osmolarity. ${ }^{14}$ Other agents such as fibrinogen mixtures ${ }^{13,14,21}$ and poloxamers ${ }^{22}$ can also be used for creating a more durable mucosa elevation. Compared with HA, fibrinogen mixtures and poloxamer solutions are significantly less expensive but remain substantially more expensive than NS. In addition, the use of fibrinogen mixtures for endoscopic resections still needs to be critically considered with regard to their potential to transfer infections.

The present research has demonstrated that duration of mucosa elevation was significantly lengthened by the use of HA at concentrations of $0.5 \%$ and $0.25 \%$, as compared with NS, a finding that agrees with other studies. However, the effect of $0.125 \%$ HA solutions on mucosa elevation was similar to NS. G, a hypertonic solution consisting of $10 \%$ glycerin with $0.9 \%$ NS plus $5 \%$ fructose, has been discussed as an alternative to HA. ${ }^{15}$ In the current study, the effect of G on mucosal elevation was inferior to HA, to the extent that no difference was observed between $\mathrm{G}$ and NS. A previous study also demonstrated the inferiority of $\mathrm{G}$ compared with $0.5 \%$ and $0.25 \% \mathrm{HA}$, but $\mathrm{G}$ did show longer-lasting elevation than NS. ${ }^{12}$ In addition, the potential role of HES was investigated and no difference was observed relative to $0.5 \%$ and $0.25 \% \mathrm{HA}$, serum, or plasma, which is consistent with the results of another recent study. ${ }^{23}$ However, there is some suggestion that HES solutions may exert adverse effects on plasmatic coagulation and platelet function, so these may promote bleeding during or after endoscopic resection. ${ }^{24}$

The results of the current study suggest that autologous blood and its components may serve as cheap alternatives for achieving a longer-lasting mucosa elevation without risk of transmission of infection. Serum and plasma seem to be as effective as $0.25 \%$ and $0.5 \%$ HA. However, a problem with the use of plasma or serum in routine endoscopy may be difficulty in rapidly preparing the patient's blood immediately prior to the endoscopic procedure, particularly because this would often need to be performed within the endoscopy unit.

Injection of whole blood gave superior height and duration of submucosa elevation compared with all other solutions tested. Therefore, autologous whole blood might serve as an optimal agent for ESD or EMR procedures that require long-lasting submucosa elevation. However, several questions have to be answered before whole blood is routinely used as a submucosal cushion fluid, as submucosal injection of autologous blood could hamper the specialist's view during the endoscopic resection procedure. Moreover, electrocauterization during endoscopic resection could impair the specialist's vision by introducing burning artifacts. However, dark dyes like methylene blue or toluidine blue are regularly used for submucosal injection without impairing vision during endoscopic resection and a recent study did not observe any impairment of visualization or other technical problems after the submucosal injection of autologous blood prior to endoscopic resection in a porcine model. Furthermore, injection of autologous blood seemed to promote local hemostasis and the clot of the fresh injected blood that formed facilitated the endoscopic resection by separating the muscularis from the submucosal layer of the gastric wall. ${ }^{25}$

Another disadvantage of using whole blood as a cushion fluid might be blood coagulation. Further studies are required to investigate whether this can be resolved by taking the blood sample immediately before submucosal injection.

Autologous blood is readily available at low cost so may represent an optimal agent for creating the mucosa elevation needed for endoscopic resection. Further in vivo studies in humans are needed to clarify the potential role of autologous blood for long-lasting EMR or ESD.

\section{Disclosure}

The authors declare no conflicts of interest in this work.

\section{References}

1. Kudo S, Kashida H, Nakajima T, Tamura S, Nakajo K. Endoscopic diagnosis and treatment of early colorectal cancer. World J Surg. 1997;21(7):694-701.

2. Ono H, Kondo H, Gotoda T, et al. Endoscopic mucosal resection for treatment of early gastric cancer. Gut. 2001;48(2):225-229.

3. Inoue H. Endoscopic mucosal resection for the entire gastrointestinal mucosal lesions. Gastrointest Endosc Clin N Am. 2001;11(3): 459-478.

4. Nakajima T. Gastric cancer treatment guidelines in Japan. Gastric Cancer. 2002;5(1):1-5.

5. Pohl J, Pech O, May A, Manner H, Ell C. Endoscopic resection of early esophageal and gastric neoplasias. Dig Dis. 2008;26(4):285-290.

6. Rosenberg N. Submucosal saline wheal as safety factor in fulguration or rectal and sigmoidal polypi. AMA Arch Surg. 1955;70(1): 120-122.

7. Tada M, Shimada M, Mrakami F, Murata H, Mizumachi S, Arima K. [Development of the strip-off biopsy.] Gastroenterol Endosc. 1984;26: 833-839. Japanese.

8. Waye JD. Saline injection colonoscopic polypectomy. Am J Gastroenterol. 1994;89(3):305-306.

9. Iishi H, Tatsuta M, Kitamura S, Narahara H, Iseki K, Ishiguro S. Endoscopic resection of large sessile colorectal polyps using a submucosal saline injection technique. Hepatogastroenterology. 1997;44(15):698-702.

10. Yamamoto H, Yube T, Isoda N, et al. A novel method of endoscopic mucosal resection using sodium hyaluronate. Gastrointest Endosc. 1999;50(2):251-256. 
11. Fujishiro M, Yahagi N, Kashimura K, et al. Different mixtures of sodium hyaluronate and their ability to create submucosal fluid cushions for endoscopic mucosal resection. Endoscopy. 2004;36(7):584-589.

12. Fujishiro M, Yahagi N, Kashimura K, et al. Comparison of various submucosal injection solutions for maintaining mucosal elevation during endoscopic mucosal resection. Endoscopy. 2004;36(7):579-583.

13. Lee SH, Park JH, Park do H, et al. Clinical efficacy of EMR with submucosal injection of a fibrinogen mixture: a prospective randomized trial. Gastrointest Endosc. 2006;64(5):691-696.

14. Hyun JJ, Chun HR, Chun HJ, et al. Comparison of the characteristics of submucosal injection solutions used in endoscopic mucosal resection. Scand J Gastroenterol. 2006;41(4):488-492.

15. Uraoka T, Fujii T, Saito Y, et al. Effectiveness of glycerol as a submucosal injection for EMR. Gastrointest Endosc. 2005;61(6):736-740.

16. Fujishiro M, Yahagi N, Nakamura M, et al. Successful outcomes of a novel endoscopic treatment for GI tumors: endoscopic submucosal dissection with a mixture of high-molecular-weight hyaluronic acid, glycerin, and sugar. Gastrointest Endosc. 2006;63(2):243-249.

17. Katsinelos P, Kountouras J, Paroutoglou G, et al. A comparative study of $50 \%$ dextrose and normal saline solution on their ability to create submucosal fluid cushions for endoscopic resection of sessile rectosigmoid polyps. Gastrointest Endosc. 2008;68(4):692-698.

18. Matsui Y, Inomata M, Izumi K, Sonoda K, Shiraishi N, Kitano S. Hyaluronic acid stimulates tumor-cell proliferation at wound sites. Gastrointest Endosc. 2004;60(4):539-543.
19. Sohn DK, Chang HJ, Choi HS, et al. Does hyaluronic acid stimulate tumor growth after endoscopic mucosal resection? J Gastroenterol Hepatol. 2008;23(8 Pt 1):1204-1207.

20. Fujishiro M, Yahagi N, Kashimura K, et al. Tissue damage of different submucosal injection solutions for EMR. Gastrointest Endosc. 2005; 62(6):933-942.

21. Lee SH, Cho WY, Kim HJ, et al. A new method of EMR: submucosal injection of a fibrinogen mixture. Gastrointest Endosc. 2004;59(2): 220-224.

22. Fernández-Esparrach G, Shaikh SN, Cohen A, Ryan MB, Thompson CC. Efficacy of a reverse-phase polymer as a submucosal injection solution for EMR: a comparative study (with video). Gastrointest Endosc. 2009;69(6):1135-1139.

23. Polymeros D, Kotsalidis G, Triantafyllou K, Karamanolis G, Panagiotides JG, Ladas SD. Comparative performance of novel solutions for submucosal injection in porcine stomachs: an ex vivo study. Dig Liver Dis. 2010;42(3)226-229.

24. Kozek-Langenecker SA. Effects of hydroxyethyl starch solutions on hemostasis. Anesthesiology. 2005;103(3):654-660.

25. Giday SA, Magno P, Buscaglia JM, et al. Is blood the ideal submucosal cushioning agent? A comparative study in a porcine model. Endoscopy. 2006;38(12):1230-1234.
Clinical and Experimental Gastroenterology

\section{Publish your work in this journal}

Clinical and Experimental Gastroenterology is an international, peerreviewed, open access journal, publishing all aspects of gastroenterology in the clinic and laboratory, including: Pathology, pathophysiology of gastrointestinal disease; Investigation and treatment of gastointestinal disease; Pharmacology of drugs used in the alimentary tract;

\section{Dovepress}

Immunology/genetics/genomics related to gastrointestinal disease. This journal is indexed on CAS. The manuscript management system is completely online and includes a very quick and fair peer-review system. Visit http://www.dovepress.com/testimonials.php to read real quotes from published authors. 